\title{
2-取代苯并噁唑类化合物合成研究进展
}

\author{
肖立伟* 高红杰 孔洁刘光仙彭晓霞 王树军
}

(廊坊师范学院化学与材料科学学院 廊坊 065000)

\begin{abstract}
摘要 2-取代苯并恶唑类化合物在医药、农药以及配位催化等领域有广阔的应用前景, 因而其合成方法备受关注. 近年 来，微波促进、水为介质、无溶剂合成、组合化学以及过渡金属催化等一系列高效、绿色的合成方法在 2-取代苯并噁 唑类化合物的合成中得到广泛的应用. 按照不同的合成原料和合成方法进行分类，综述了近年来 2-取代苯并噁唑类化 合物合成研究的新进展.
\end{abstract}

关键词 2-取代苯并噁唑; 邻氨基苯酚; 偶联反应; 过渡金属催化; 绿色化学

\section{Progress in the Synthesis of 2-Substituted Benzoxazoles Derivatives}

\author{
Xiao, Liwei* Gao, Hongjie \\ Kong, Jie \\ Liu, Guangxian \\ Peng, Xiaoxia \\ Wang, Shujun \\ (Faculty of Chemistry and Material Science, Langfang Teachers University, Langfang 065000)
}

\begin{abstract}
Substituted benzoxazole derivatives play important roles in pharmaceuticals, pesticide and coordination catalysis, so the synthetic methods for 2-substituted benzoxazoles have been attracted widely. Recently, a series of efficient green methods such as microwave irradiation, water as solvent, solvent-free, combinatorial chemistry and transition metal catalysis etc. have been used to synthesize 2-substituted benzoxazoles derivatives. Based on different starting materials and different methods, the recent advances in synthesis of 2-substituted benzoxazole are reviewed.
\end{abstract}

Keywords 2-substituted benzoxazoles; $o$-aminophenol; coupled reaction; transition metal catalysis; green chemistry

苯并噁唑类化合物是一种分子结构中含有氮原子 和氧原子的苯并杂环化合物. 该类化合物表现出良好的 抗菌、消炎、杀灭植物病毒功效 ${ }^{[1]}$ 和配位性能 ${ }^{[2]}$, 在医药、 农药以及配位催化领域应用广泛. 苯并噁唑骨架连接的 取代基的类型和位置对其性质有较大影响，通过构效关 系研究发现，2-位上取代基的类型对其生理活性的影响 最大 ${ }^{[3]}$. 因此, 2-取代苯并噁唑类化合物的合成方法受 到人们的广泛关注 ${ }^{[4]}$.

选择合适的原料和成环方法是合成 2-取代苯并噁 唑类化合物的关键步骤. 邻氨基苯酚能够与醇、卤代烃、 醛、羧酸及其衍生物等反应生成噁唑杂环, 是合成 2-取 代苯并噁唑最常用的原料; 此外, 近年来, 苯并噁唑与 含不同功能基团的化合物通过偶联反应合成 2-取代苯 并噁唑的报道逐年增多. 基于此, 本文按不同的合成原 料和方法进行分类, 从 6 个方面对近年来 2-取代苯并噁
坐类化合物的合成方法进行了综述和展望.

\section{1 以邻氨基苯酚为原料合成 2-取代苯并噁唑}

\section{1 邻氨基苯酚与醛反应合成 2-取代苯并噁唑}

邻氨基苯酚是合成 2-取代苯并噁唑最常用的原料 之一. 通常利用氧气、杂多酸、分子碘以及高价金属氧 化剂等为催化剂促使邻氨基苯酚与醛反应生成 2-取代 苯并噁唑. 例如, Kawashita 等 ${ }^{[5]}$ 发现, 在活性炭负载氧 气的作用下, 邻氨基苯酚与芳醛发生缩合反应生成 2-芳 基苯并噁唑(Eq. 1), 产率为 $67 \% \sim 88 \%$. 研究发现, 当 醛中含有吸电子基团时产率明显比含有供电子基团时 要高.

整个反应过程分为两个阶段进行: 首先邻氨基苯酚 中的氨基与芳醛发生缩合反应生成 Schiff碱, Schiff碱经 过环化反应生成 2-芳基苯并噁唑啉，随后在氧化体系

\footnotetext{
*E-mail: xiaoliwei2000@sina.com

Received January 19, 2014; revised February 20, 2014; published online March 12, 2014.

Project supported by the Natural Science Foundation of Hebei Province (No. B2013408014) and the Key Foundation of Langfang Teachers University (No. LSZZ201302).

河北省自然科学基金(No. B2013408014)和廊坊师范学院重点基金(No. LSZZ201302)资助项目.
} 


$$
\mathrm{R}^{1}=\mathrm{H}, \mathrm{CH}_{3}, \mathrm{NO}_{2} ; \mathrm{R}^{2}=\mathrm{H}, \mathrm{CH}_{3}, \mathrm{OCH}_{3}, \mathrm{Cl}, \mathrm{CN}, \mathrm{NO}_{2}
$$

$\operatorname{DarcoKB} / \mathrm{O}_{2}$ 的作用下脱氢形成 2-芳基苯并噁唑(Scheme 1). 该方法选用 $\mathrm{O}_{2}$ 为氧化剂, 与采用金属和有机试剂为 催化剂的类似反应相比，成本低、环境友好、产率高.

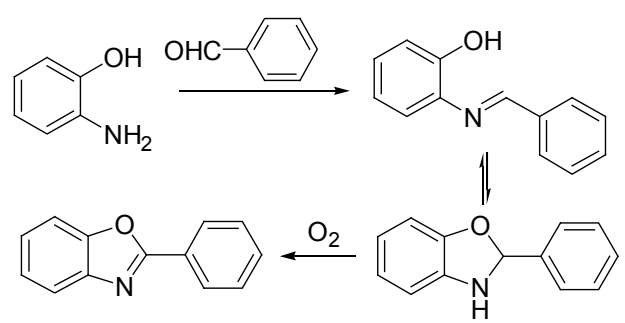

Scheme 1

2007 年, Haneda 等 ${ }^{[6]}$ 采用上述方法, 以邻氨基苯酚 与吡啶醛为原料, 合成了 2-(2-吡啶)苯并噁唑(Eq. 2), 产 率可达 $86 \%$. 以 2-(2-吡啶)苯并噁唑为配体的钯配合物, 具有较好的抗癌活性.

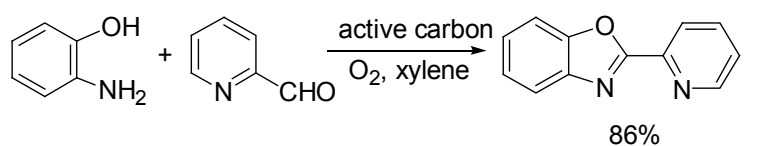

2011 年, $\mathrm{Xiao}^{[7]}$ 在偏钒酸铵催化下, 以邻氨基苯酚 和芳醛为原料, 在室温条件下合成了一系列 2-芳基苯并 噁唑(Eq. 3). 我们研究了不同溶剂对反应的影响, 结果 发现强极性的质子溶剂乙醇是该反应的理想溶剂. 偏钒 酸铵之所以有较好的催化作用, 主要是因为醛羰基氧原 子上的孤电子对填充到偏钒酸铵的钒原子空 $\mathrm{d}$ 轨道中, 使羊基活化，加速了反应的进行. 芳醛上所连基团对反 应有较大影响, 当芳环上连有吸电子基团(如 $\mathrm{NO}_{2}$ ) 时, 产率比芳环上连有供电子基团(如 $\mathrm{CH}_{3}$ ) 时相对要高些. 该方法的特点是操作简便、反应条件温和, 产率高.

$\mathrm{Cao}$ 等 $^{[8]}$ 发现氯化铁是合成 2-取代苯并噁唑的有效 催化剂. 在 $\mathrm{O}_{2}$ 气氛中, 邻氨基苯酚与芳醛在 $\mathrm{FeCl}_{3}$ 作用 下合成 2-取代苯并噁唑(Eq. 4), 产率最高可达 90\%. 在 反应中引入了串联氧化的理念, 利用氧气氧化反应体系 中的低价铁到高价铁, 继续参与到反应中来, 符合绿色 化学的理念. 该方法也可以用于 2-取代苯并噻唑和 2-取 代苯并咪唑的合成.

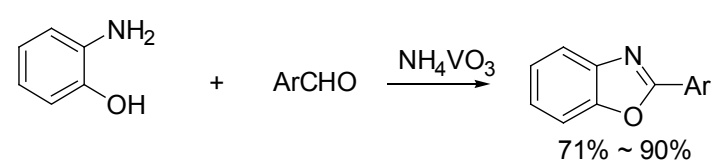

$\mathrm{Ar}=\mathrm{C}_{6} \mathrm{H}_{5}, 4-\mathrm{CH}_{3} \mathrm{C}_{6} \mathrm{H}_{4}, 4-\mathrm{ClC}_{6} \mathrm{H}_{4}, 4-\mathrm{H}_{2} \mathrm{NC}_{6} \mathrm{H}_{4}$, etc.

\begin{tabular}{clc||clc}
\hline Entry & \multicolumn{1}{c}{$\mathrm{Ar}$} & Yield/\% & Entry & \multicolumn{1}{c}{$\mathrm{Ar}$} & Yield/\% \\
\hline 1 & $\mathrm{Ph}$ & 83 & 6 & $4-\mathrm{H}_{2} \mathrm{NC}_{6} \mathrm{H}_{4}$ & 78 \\
2 & $4-\mathrm{CH}_{3} \mathrm{C}_{6} \mathrm{H}_{4}$ & 80 & 7 & $4-\mathrm{CNC}_{6} \mathrm{H}_{4}$ & 88 \\
3 & $4-\mathrm{CH}_{3} \mathrm{OC}_{6} \mathrm{H}_{4}$ & 84 & 8 & $4-\mathrm{NO}_{2} \mathrm{C}_{6} \mathrm{H}_{4}$ & 90 \\
4 & $4-\mathrm{ClC}_{6} \mathrm{H}_{4}$ & 82 & 9 & 2- $\mathrm{Pyridyl}_{4}$ & 71 \\
5 & $4-\mathrm{BrC}_{6} \mathrm{H}_{4}$ & 80 & & & \\
\hline
\end{tabular}

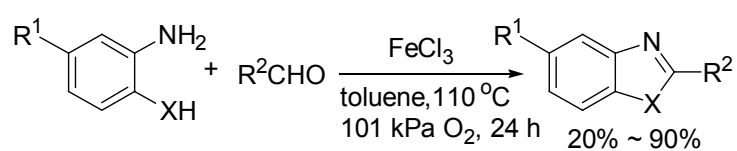

$$
\begin{aligned}
& \mathrm{X}=\mathrm{O}, \mathrm{NH}, \mathrm{S} ; \mathrm{R}^{1}=\mathrm{H}, \mathrm{CH}_{3}, \mathrm{Cl} \\
& \mathrm{R}^{2}=\mathrm{Ph}, p-\mathrm{MeC}_{6} \mathrm{H}_{4}, p-\mathrm{ClC}_{6} \mathrm{H}_{4}, \text { 1-naphthyl, 3-thienyl, 4-pyridyl }
\end{aligned}
$$

硅胶负载型催化剂由于催化效率高, 可循环使用, 备受人们的青睐. Inamdar 等 ${ }^{[9]}$ 以硅胶负载纳米氧化铜 $\left(\mathrm{CuO} / \mathrm{SiO}_{2}\right)$ 为催化剂, 催化邻氨基苯酚与各种醛反应生 成相应的 2-取代苯并噁唑(Eq. 5). 结果表明, 芳香醛的 产率明显高于脂肪醛. 该方法反应条件温和, 快速高效, 克服了传统试剂价格昂贵、腐蚀性强以及需要添加剂等 缺点, 而且该方法还可以用于合成 2-取代苯并噻唑和 2取代苯并咪唑.

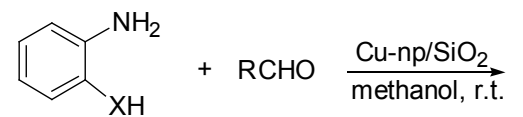

$\mathrm{X}=\mathrm{NH}, \mathrm{S}, \mathrm{O}$

$\mathrm{R}=$ Aromatic, Heteroaromatic, Aliphatic

Shavaleev 等 ${ }^{[10]}$ 以邻氨基苯酚和 6-着圣甲基-吡啶-2甲醛为原料, 在二噁烷和乙醇中反应合成了 2-取代苯并 啞唑发光材料(Eq. 6), 该材料可用于分辨活细胞的苂光 成像方面.<smiles>O=Cc1cccc(CO)n1</smiles><smiles>[R]c1ccc(O)c(N)c1</smiles>

$$
\begin{aligned}
& \text { (i) dioxane/ethanol } \\
& \mathrm{N}_{2}, 110^{\circ} \mathrm{C} \\
& \text { (ii) } \mathrm{DDQ}, \mathrm{CH}_{2} \mathrm{Cl}_{2}, \mathrm{~N}_{2} \\
& \text { sonication }
\end{aligned}
$$<smiles>OCc1cccc(-c2nc3ccccc3o2)n1</smiles>

杂多酸是近年来在有机合成中研究较多的催化剂. 如 Heravi 等 ${ }^{[11]}$ 以邻氨基苯酚和苯甲醛为原料, 在催化量 的杂多酸 $\mathrm{H}_{5}\left[\mathrm{PMo}_{10} \mathrm{~V}_{2} \mathrm{O}_{40}\right], \mathrm{H}_{4}\left[\mathrm{PMo}_{11} \mathrm{VO}_{40}\right]$ 和 $\mathrm{H}_{3}\left[\mathrm{PMo}_{12}-\right.$ $\mathrm{O}_{40}$ 的作用下, 采用一锅法合成了 2-芳基苯并噁唑(Eq. 
7), 该方法产物易提纯，收率最高可达到 95\%.<smiles>Nc1ccccc1O</smiles>

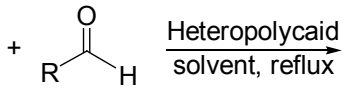

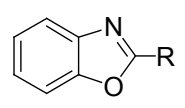

$77 \% \sim 95 \%$
$\mathrm{R}=\mathrm{Ph}, 4-\mathrm{MeC}_{6} \mathrm{H}_{4}, 4-\mathrm{NO}_{2} \mathrm{C}_{6} \mathrm{H}_{4}, 4-\mathrm{MeOC}_{6} \mathrm{H}_{4}$

近年来, 高价碘试剂作为一种性能温和、环境友好 的氧化剂在有机合成中得到了广泛的应用. 2009 年, Kumar 等 ${ }^{[12]}$ 以聚苯乙烯固载高价碘试剂为催化剂, 邻氨 基苯酚和芳醛在室温下反应 5 10 min, 生成 2-取代苯 并噁唑(Eq. 8), 产率最高可达 96\%. 该方法也同样适用 于 2-取代苯并噻唑和 2-取代苯并咪唑的合成.

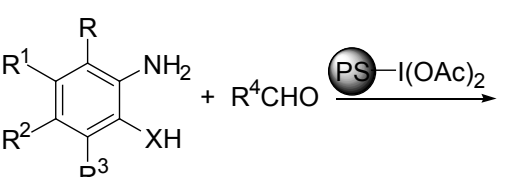

$X=\mathrm{NH}, \mathrm{O}, \mathrm{S}$<smiles>[R][X]1c([R])nc2c([R])c([R])c([R])c([R])c12</smiles>

$85 \% \sim 96 \%$
Firouz 等 ${ }^{[13]}$ 则利用单质碘催化, 在室温条件下合成 了 2-取代苯并噁唑, 产率为 $80 \%$. 该方法条件温和, 不 需要溶剂, 是一绿色环保的合成方法.

\section{2 邻氨基苯酚与羧酸反应合成 2-取代苯并噁唑}

Balaswamy 等 ${ }^{[14]}$ 通过带取代基的邻氨基苯酚在甲 酸、乙酸或丙酸中回流 $4 \mathrm{~h}$, 合成了相应的 2-取代苯并 啞唑(Eq. 9). 该方法加入过量的液态羧酸做为溶剂, 不 需要任何催化剂, 操作简单.

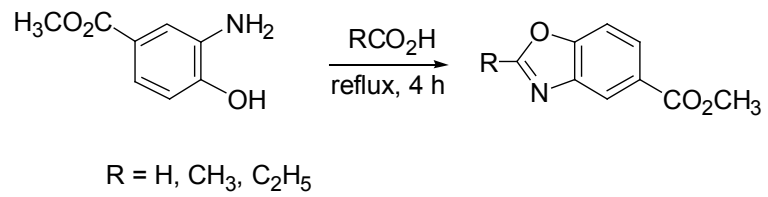

多聚磷酸(PPA)催化效果好, 使用安全, 反应过程 中无炭化现象, 反应后处理简单, 因而受到人们重视. 2008 年, Ertan 等 ${ }^{[15]}$ 在 PPA 催化下, 取代邻氨基苯酚与 羧酸作用生成 2-芳基取代苯并噁唑(Eq. 10), 产率为 45\% 85\%. 取代基对反应影响较大, 邻氨基苯酚中有 硝基时产率最大 $(85 \%)^{[16]} .2010$ 年, Soares 等 ${ }^{[17]}$ 在 PPA 催 化下, 以溴乙酸和邻氨基苯酚为原料合成了 2 -溴甲基苯

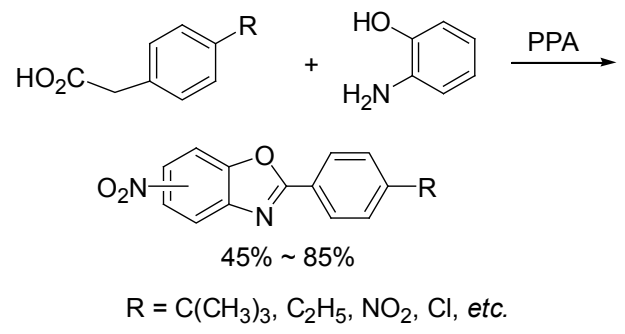

并噁唑，产率为 $58 \%$.

微波辐射具有效率高、操作方便等优点，已经在有 机合成中得到广泛的应用. 2003 年，周红军等 ${ }^{[18]}$ 在微波 辐射和多聚磷酸作用下, 邻氨基苯酚和吡啶二䧳酸反应 得到 2,6-二苯并啞唑基吡啶(Eq. 11), 产率为 76\%.整个 反应过程只需 $4 \mathrm{~min}$, 与传统加热方法相比, 效率大大 提高.

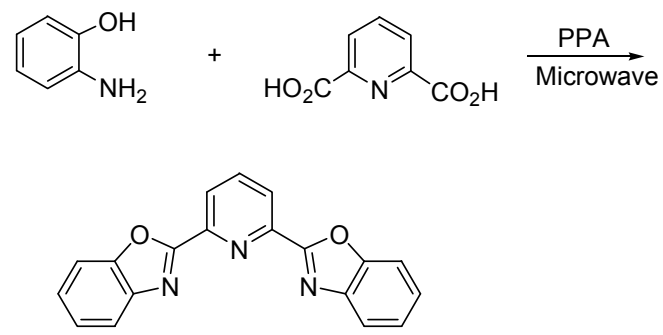

Heravi 等 ${ }^{[19]}$ 以杂多酸(HPAs)为催化剂, 以邻氨基苯 酚与芳酸为原料合成了 2-芳基苯并噁唑(Eq. 12), 产率 为 $78 \% \sim 95 \%$. 反应结束后，杂多酸经过简单处理，还 可以重复使用.

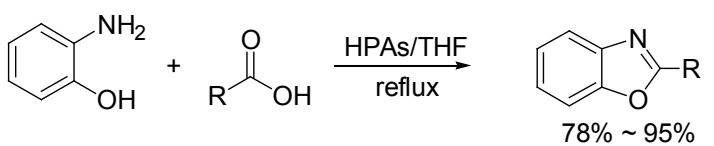

$\mathrm{R}=\mathrm{Ph}, 4-\mathrm{MeC}_{6} \mathrm{H}_{4}, 4-\mathrm{NO}_{2} \mathrm{C}_{6} \mathrm{H}_{4}, 4-\mathrm{OMeC}_{6} \mathrm{H}_{4}$

\section{3 邻氨基苯酚与羧酸衍生物反应合成 2-取代苯并噁 唑}

固体酸是催化邻氨基苯酚与酯反应合成 2-取代苯 并噁唑的良好催化剂. 2010 年, Boyle 等 ${ }^{[20]}$ 以对甲苯磺酸 $(p-\mathrm{TsOH})$ 为催化剂, 邻氨基苯酚与 $(\mathrm{MeO})_{3} \mathrm{CCO}_{2} \mathrm{Me}$ 作用 得到 2-甲酸甲酯基苯并噁唑(Eq. 13)，产率达 $87 \%$. 该化 合物是合成抗菌、抗癌药物多卡米星的中间体. 他们认 为该反应的机理是，酸性催化剂促使邻氨基苯酚中的着全 基和氨基与酯发生酯交换反应，脱去醇分子成环形成目 标产物.

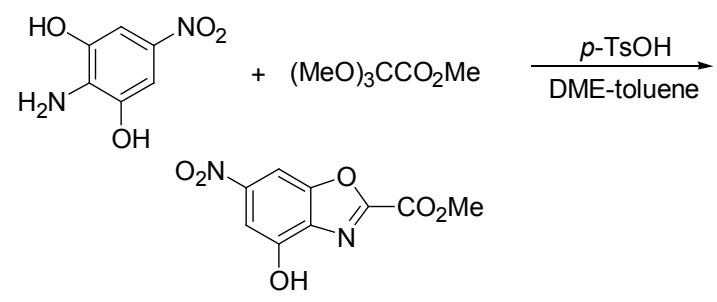

Radi 等 ${ }^{[21]}$ 以对甲苯磺酸为催化剂，在微波辐射下， 采用一锅法反应合成了一系列 2-取代苯并噁唑化合物 (Eq. 14). 该方法反应时间短，产率最高可达到 $99 \%$. 


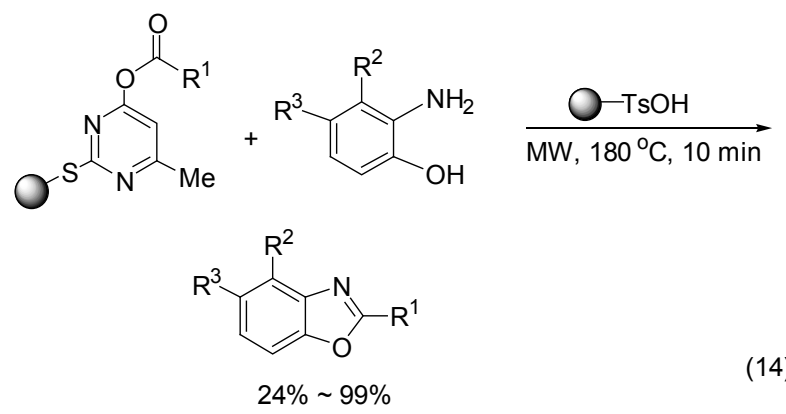

$\mathrm{R}^{1}=4-\mathrm{ClC}_{6} \mathrm{H}_{4}, 2-\mathrm{FC}_{6} \mathrm{H}_{4}$, etc.; $\mathrm{R}^{2}=\mathrm{H}, \mathrm{NO}_{2} ; \mathrm{R}^{3}=\mathrm{H}, \mathrm{Cl}, \mathrm{Me}$

2011 年, 我们 ${ }^{[2]}$ 以 $\beta$-酮酯与邻氨基苯酚为原料, 在 对甲苯磺酸催化下，合成一系列 2-取代苯并噁唑(Eq. $15)$, 产率为 $26 \% \sim 79 \%$. 该方法反应条件温和, 原料中 的一些功能基团在反应过程中不受影响, 提供了一种合 成带有多官能团的杂环化合物的新方法. 该方法同样适 用于 2-取代苯并咪唑的合成.

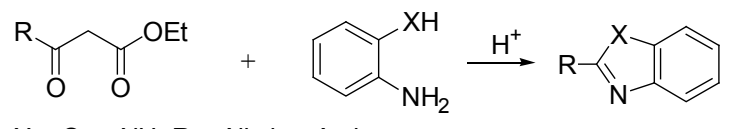

$$
\mathrm{X}=\mathrm{O} \text { or } \mathrm{NH} ; \mathrm{R}=\text { Alkyl or Aryl }
$$

该反应的历程可以描述如下：首先，在酸性条件下， 氨基进攻 $\beta$ 碳上的羰基, 失去一分子水形成中间体 $\mathbf{a}$ 与 $\mathbf{b}$; 随后, 化合物 $\mathbf{b}$ 上的羟基进攻 $\beta$ 羰基发生 Michael 加 成反应, 失去 1 分子乙酸乙酯, 形成 2-取代苯并噁唑 (Scheme 2). 生成 2-取代苯并咪唑的反应机理与之相似.

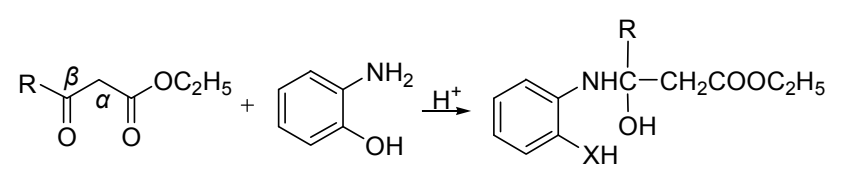

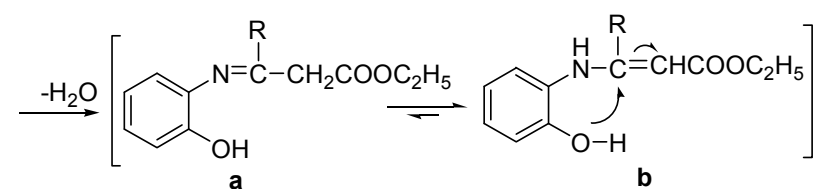<smiles>CCOC(=O)OCc1nc2ccccc2o1</smiles>

Scheme 2

硅胶负载硫酸不但保持了硫酸成本低廉、催化效率 高的优点, 而且克服了硫酸的强腐蚀性和氧化性等缺 点, 因而倍受人们的青崃. Mohammadpoor-Baltork 等 ${ }^{[23]}$ 采用硅胶负载硫酸为催化剂, 三烷基酸酯和邻氨基苯酚 在无溶剂条件下反应生成 2-甲基苯并㤎唑(Eq. 16), 产 率最高可达 $97 \%$. 反应完成后, 催化剂硅胶负载硫酸经 过简单清洗, 可以重复使用. 该方法也适用于合成 2-取 代苯并咪唑.

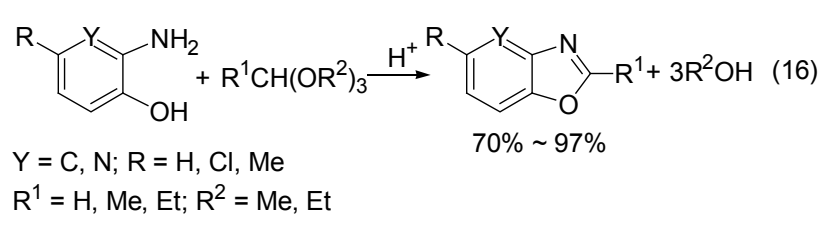

固相合成是近年来快速发展起来的一种合成方法, 在有机合成、药物合成等领域受到越来越多的关注. 2008 年, Lim 等 ${ }^{[24]}$ 在微波辐射和 PPA 催化下, 利用树脂 固载化的酯与邻氨基苯酚作用合成了一系列 2-取代苯 并噁唑(Eq. 17), 产率最高可达 96\%.

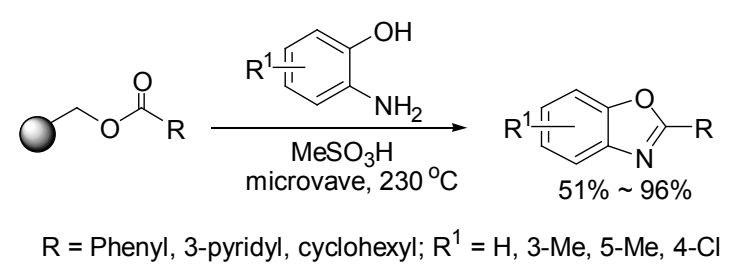

除质子酸外, Lewis 酸也可以催化上述反应. Bastug 等 ${ }^{[25]}$ 在室温条件下, 邻氨基苯酚与功能化的原酸酯在 $\mathrm{BF}_{3} \cdot \mathrm{OEt}_{2}$ 的 $\mathrm{CH}_{2} \mathrm{Cl}_{2}$ 溶液中反应, 生成 2-取代苯并噁唑 (Eq. 18), 产率为 $75 \% \sim 95 \%$. 该方法操作简单，反应条 件温和，在反应过程中，原料分子上携带的功能基团不 受影响, 为功能化的杂环化合物提供了新的合成方法. 该方法也同样适用于 2-取代苯并噻唑和 2-取代苯并咪 唑的合成.

$$
\begin{aligned}
& \mathrm{X}=\mathrm{NH}, \mathrm{O}, \mathrm{S} ; \mathrm{R}=\mathrm{Cl}, \mathrm{Br}, \mathrm{CH}_{3}, \mathrm{CO}_{2} \mathrm{Me}, \mathrm{H} \\
& \mathrm{R}^{1}=\left(\mathrm{CH}_{2}\right)_{n} \mathrm{X},\left(\mathrm{CH}_{2}\right)_{n} \mathrm{CH}=\mathrm{CH}_{2},\left(\mathrm{CH}_{2}\right)_{n} \mathrm{CN} ; \mathrm{R}^{2}, \mathrm{R}^{3}=\text { alkyl }
\end{aligned}
$$

邻氨基苯酚不但与酯反应生成 2-取代苯并噁唑, 也 能与腈发生类似反应. Jeyanthi 等 ${ }^{[26]}$ 以腈类化合物与邻 氨基苯酚为原料, 在浓盐酸中回流 $6 \mathrm{~h}$, 合成了一种 2取代苯并噁唑类衍生物(Eq. 19). 该物质具有较强的生 理活性, 是合成胃肠疾病药物的重要中间体.<smiles>[R]Cc1ccc(C(C#N)n2cnc3cccc([R])c32)cc1</smiles>

该反应经历如下历程: 首先, 在酸性条件下, 腈与 $\mathrm{HCl}$ 发生加成反应生成亚胺化合物 $\mathbf{d}, \mathbf{d}$ 与邻氨基苯酚中 的羟基作用生成化合物 $\mathbf{e}$, 最后经环化缩合，脱去一分 子氨生成苯并噁唑 f (Scheme 3). 因为反应在强酸性环 境中发生, 生成的氨被酸吸收, 大大促进了反应的进行. 
<smiles>[R]c1ccc(C(C#N)n2cnc3ccccc32)cc1</smiles><smiles>[R]c1ccc(C(C(=N)Cl)n2cnc3ccccc32)cc1</smiles><smiles>Nc1ccccc1O</smiles>

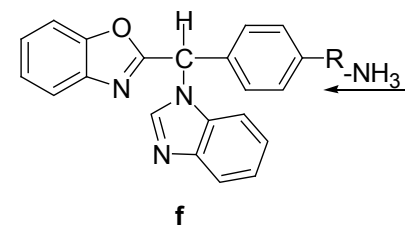<smiles>[R]c1ccc(C(C)(N)C(=N)Oc2ccccc2N)cc1</smiles>

Scheme 3

2012 年, Christina 等 ${ }^{[27]}$ 以硫氰化合物与邻氨基苯酚 为原料, 在 $\mathrm{CS}_{2}$ 中加热到 $160{ }^{\circ} \mathrm{C}$, 生成 2-硫代苯并噁唑 (Eq. 20), 产率为 $61 \% \sim 86 \%$. 所得产物具有良好的抗 菌、消炎、镇痛的功效.<smiles>[R]c1c(SC#N)ccc(N([R])[R])c1[R]</smiles><smiles>[R]c1c(Sc2nc3ccccc3o2)ccc(N([R])[R1])c1[R]</smiles>

$\mathrm{R}^{1}, \mathrm{R}^{2}=\mathrm{H}, \mathrm{NO}_{2}, \mathrm{COOH} ; \mathrm{R}^{3}, \mathrm{R}^{4}=\mathrm{H}, \mathrm{C}_{2} \mathrm{H}_{5}$

邻氨基苯酚还能与酰氯反应生成 2-取代苯并噁唑. 2002 年, Pottorf 等 ${ }^{[28]}$ 在微波辐射条件下, 以邻氨基苯酚 和酰氯为原料合成了 2-芳基苯并啞唑(Eq. 21). 该反应 分两步完成, 首先邻氨基苯酚和酰氯作用生成邻酯基酰 胺，随后关环生成 2-芳基苯并噁唑. 该方法操作简便, 在反应中没有副产物产生. 当邻氨基苯酚含有供电子基 团、酰氯含有吸电子基团时，产率最高可达 $98 \%$.

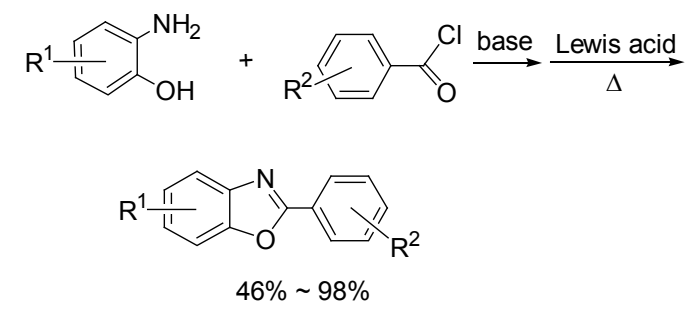

$\mathrm{R}^{1}=\mathrm{CH}_{3}, \mathrm{NO}_{2}, \mathrm{EtS}, \mathrm{C}\left(\mathrm{CH}_{3}\right)_{2} \mathrm{Et} ; \mathrm{R}^{2}=\mathrm{Br}, \mathrm{Ph}, \mathrm{NO}_{2}, \mathrm{OMe}$

介孔材料是近些年发展起来的一种新型的无机材 料, 具有较好物理和化学稳定性, 在分离、催化等领域 有广泛的应用. Shoar 等 ${ }^{[29]}$ 以介孔材料 MCM-41 为催化 剂, 邻氨基苯酚与苯甲酰氯反应合成了 2-芳基苯并噁唑
(Eq. 22), 收率最高可达 90\%. MCM-41 规则的孔道效应 增大了催化剂与反应底物间的接触面积，对反应有很好 的促进作用.
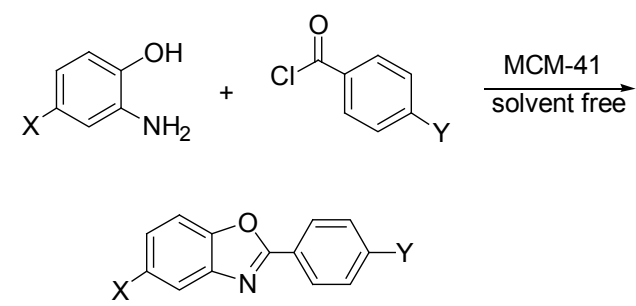

2004 年, Nadaf 等 ${ }^{[30]}$ 在醋酸碘苯催化下, 通过邻氨 基苯酚与酰氯作用高效率地合成了 2-苯基苯并噁唑(Eq. 23). 该方法反应条件温和，后处理简单，是合成 2-取代 苯并噁唑的一种理想方法.

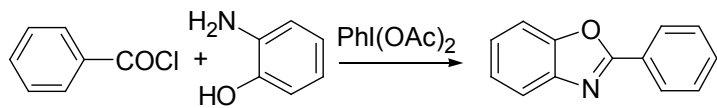

Chun 等 ${ }^{[31]}$ 在二甲苯中, 以嗍酸为催化剂, 通过酰 氯与邻氨基酚作用, 生成 2-取代苯并噁唑(Eq. 24), 产率 为 $63 \%$.

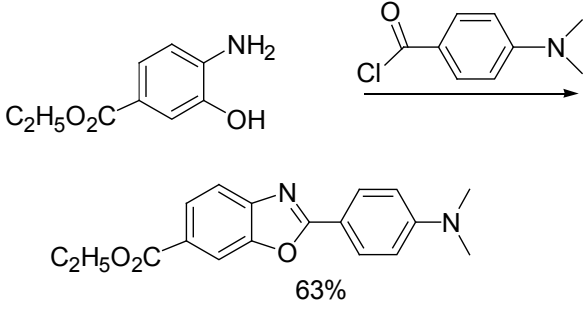

\section{4 邻氨基苯酚与芳甲基反应合成 2-取代苯并噁唑}

2008 年, Gao 等 ${ }^{[32]}$ 以邻氨基苯酚与 2-甲基吡啶类化 合物为原料, 在单质硫存在下，在 $170{ }^{\circ} \mathrm{C}$ 反应合成了 2 吡啶基苯并噁唑(Eq. 25), 产率为 28\% 37\%. 该化合物 与镍形成的配合物对乙烯低聚具有良好的催化活性. 该 方法打开了一条由烃基合成 2-取代苯并噁唑的简洁途 径.

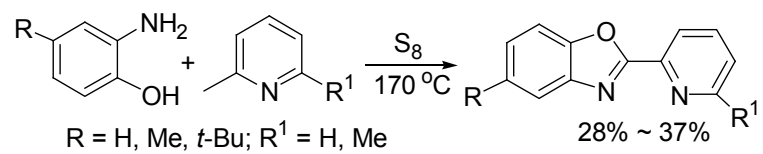

串联氧化体系是一种二元氧化体系，一般由催化量 的贵重或稀有氧化剂和化学计量的常用氧化剂组成, 广 泛应用于有机合成中. 2013 年, $\mathrm{Gu}$ 等 $^{[33]}$ 在过氧化二叔丁 基与 $\mathrm{FeBr}_{2}$ (DTBP-FeBr$)_{2}$ 组成的串联氧化体系中, 通过 邻氨基苯酚与甲苯及其衍生物作用，羟基、氨基和甲基

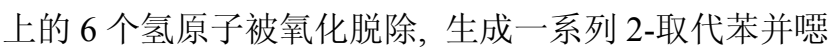
唑(Eq. 26), 产率为 36\% 79\%. 


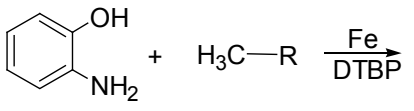

$\mathrm{R}=$ aryl, heteroaryl

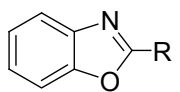

$36 \% \sim 79 \%$

\section{5 邻氨基苯酚与醇反应合成 2-取代苯并噁唑}

Wilfred 等 ${ }^{[34]}$ 以邻氨基苯酚和苠醇为原料, 在二氧 化锰催化下，生成 2-苯基苯并噁唑(Eq. 27).<smiles>Nc1ccccc1OCc1nc2ccccc2o1</smiles>

该反应经历一个连续氧化的历程: 首先, 苠醇在 $\mathrm{MnO}_{2}$ 作用下生成苯甲醛, 苯甲醛再与邻氨基苯酚缩合 生成五元环中间体, 进而在 $\mathrm{MnO}_{2}$ 作用下脱氢生成 2-苯 基苯并噁唑(Scheme 4). 该方法反应条件温和, 简单快 速，可以由醇直接合成 2-取代苯并噁唑、2-取代苯并咪 唑、2-取代苯并噻唑类化合物.

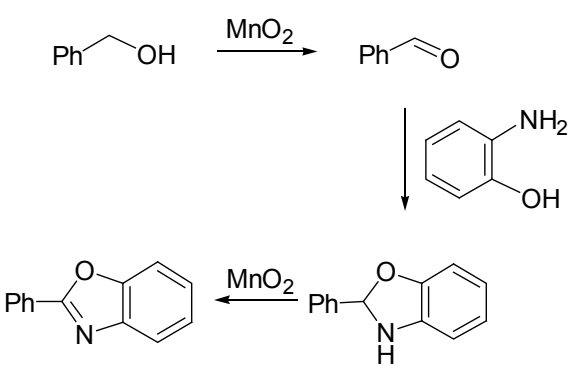

Scheme 4

$\mathrm{Wu}$ 等 ${ }^{[35]}$ 以邻硝基苯酚和茮醇为原料, 在 $\mathrm{FeCl}_{3}$, $\mathrm{FeCl}_{2}$ 或 1,1'-双(二苯基膦)二茂铁(dppf)等催化下生成 2取代苯并噁唑(Eq. 28), 产率最高可达 $89 \%$. 该反应的历 程为：首先，在 $\mathrm{FeCl}_{3}, \mathrm{FeCl}_{2}$ 等作用下，邻硝基苯酚还原 为邻氨基苯酚，而茮醇则被氧化为芳醛；随后，邻氨基 苯酚与芳醛经过缩合一环化过程形成五元环中间体, 进 一步脱氢生成 2-芳基苯并噁唑. 该反应中醇的氧化、硝 基还原、缩合以及脱氢过程巧妙地串联进行, 无需外加 其他还原剂和氧化剂。

$$
\mathrm{R}^{1}=\mathrm{CH}_{3}, \mathrm{OMe}, \mathrm{F}, \mathrm{Cl}, \mathrm{Br}
$$

\section{6 邻氨基苯酚与伯胺反应合成 2-取代苯并噁唑}

2012 年, Endo 等 ${ }^{[36]}$ 在 $\mathrm{Ru}$ 催化体系作用下, 通过邻 氨基苯酚与茮胺作用生成 2-苯基苯并啞唑(Eq. 29), 产 率最高可达 $92 \%$. 整个反应过程条件温和, 催化剂在反 应中可以再生, 是合成化学未来发展的一个重要方向. 该方法的适用范围还可进一步扩展到 2-取代苯并咪唑
和 2-取代苯并噻唑的合成.
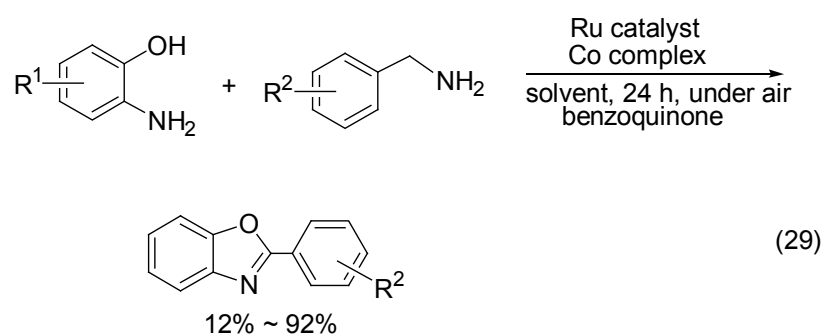

$\mathrm{R}^{1}=\mathrm{F}, \mathrm{Cl}, \mathrm{Br}, t-\mathrm{Bu}, \mathrm{Me} ; \mathrm{R}^{2}=\mathrm{C}_{6} \mathrm{H}_{4} \mathrm{Me}, \mathrm{C}_{6} \mathrm{H}_{4} \mathrm{OMe}$

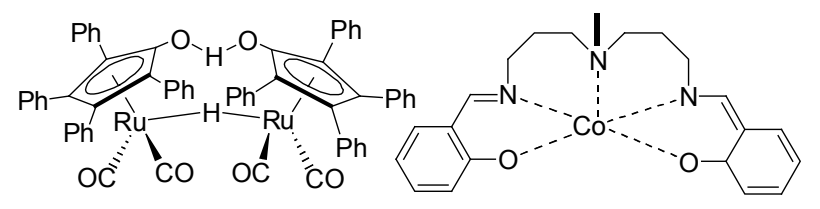

Ru catalyst

Co complex<smiles>COC1=CC(=O)C=C(OC)C1=O</smiles>

benzoquinone

该反应的机理如下：首先，芐胺被钓催化剂氧化为 芐亚胺，而钓催化剂则被还原为低价态钓配合物; 随后， 芐亚胺与邻氨基酚经过缩合-环化得到五元环中间体, 并在钓催化剂作用下脱氢得 2-苯基苯并噁唑; 还原态钉 配合物被醌重新氧化为钓催化剂, 循环使用, 而醌则被 还原为相应的酚; 酚在钴配合物催化下被空气氧化为 醌，继续参与反应(Scheme 5).

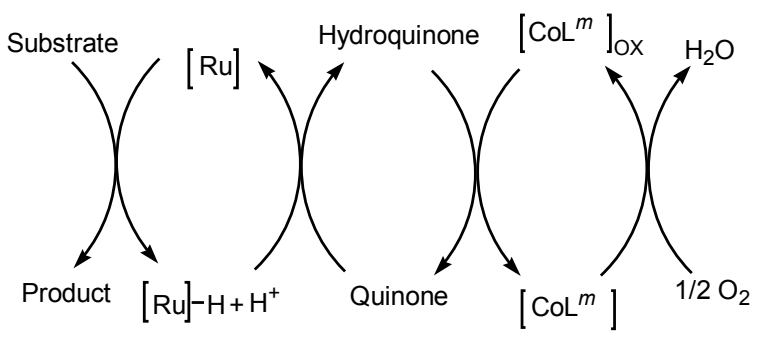

\section{Scheme 5}

2013 年, Jin 等 ${ }^{[37]}$ 在 $\mathrm{Pd} / \mathrm{C}$ 的催化作用下，邻氨基苯 酚和取代苯乙胺在二甲基乙酰胺(DMA)中，通过脱氢生 成 2-芳基苯并噁唑和氢气(Eq. 30), 产率为 70\% 92\%.

2012 年, Nguyen 等 ${ }^{[38]}$ 在痕量单质硫的存在下, 通过 邻氨基苯酚与脂肪胺作用合成了 2-取代苯并噁唑(Eq. 31 ), 产率为 $43 \% \sim 80 \%$. 上述反应过程在无溶剂和无其 他催化剂的条件下进行, 符合绿色化学的理念. 
<smiles>Nc1ccccc1O</smiles><smiles>[R]c1ccc(CN)cc1</smiles>

$$
\mathrm{R}=\mathrm{H}, \mathrm{F}, \mathrm{CH}_{3}, \mathrm{OCH}_{3}
$$

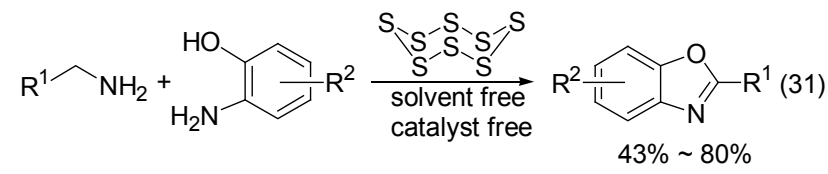

$\mathrm{R}^{1}=\mathrm{Me}, \mathrm{Ph}$, pyridine $; \mathrm{R}^{2}=\mathrm{Me}, \mathrm{Cl}$

该反应的历程为, 芐胺与硫作用生成硫代苯甲酰胺 中间体，同时放出硫化氢; 硫代苯甲酰胺中间体与邻氨 基酚作用生成邻羟基硫代苯甲酰胺基苯, 再经环化脱去 硫化氢形成 2-苯基苯并噁唑(Scheme 6).
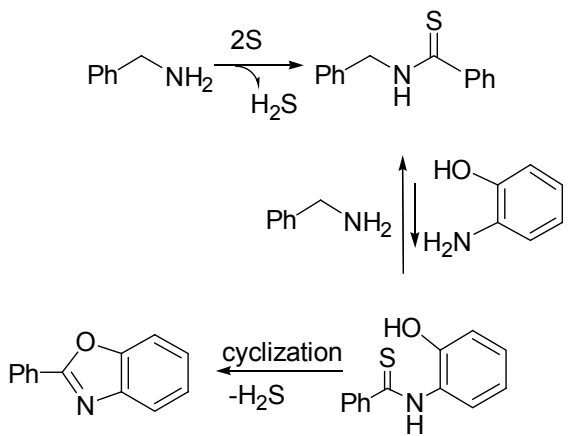

\section{Scheme 6}

\section{7 邻氨基苯酚与其他物质反应合成 2-取代苯并噁唑}

Boissarie 等 ${ }^{[39]}$ 采用芳基卤化物、异氧化物和邻氨基 苯酚三组分一锅法, 在 $\mathrm{Pd}(\mathrm{dppf}) \mathrm{Cl}_{2}-\mathrm{Cs}_{2} \mathrm{CO}_{3}$ 体系催化下, 合成了 2-取代苯并噁唑(Eq. 32). 该方法简单易行, 催化 效率高，而且用异氰化物代替有毒的 $\mathrm{CO}$ 气体，避免了 环境污染.

$$
\begin{aligned}
& \text { Xoluene, reflux } \\
& \mathrm{R}^{1}=\mathrm{OMe}, \mathrm{Ph} ; \mathrm{R}^{2}=\mathrm{Cl}, \mathrm{CH}_{3}
\end{aligned}
$$

张成仁等 ${ }^{[40]}$ 以邻氨基苯酚为原料, 选用 $\mathrm{CS}_{2}$ 作为碳 源及硫源，在 $\mathrm{KOH}$ 作用下环合得到 2-颈基苯并噁唑, 进一步与 2-氯-5-氯甲基吡啶作用，生成具有抗肿瘤活 性的 2-氯-(6-氯吡啶-3-甲基硫代)苯并噁唑(Scheme 7), 产率为 $64.7 \%$.
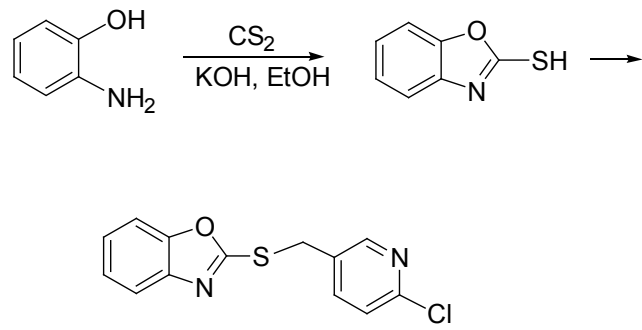

Scheme 7

\section{2 经由取代酰胺基苯合成 2-取代苯并噁唑}

\section{1 由邻卤代酰胺基苯合成 2-取代苯并噁唑}

由邻卤代酰胺合成 2-取代苯并噁唑一般在铜、钯等 过渡金属催化下完成, 在催化过程中往往需要二胺类配 体的参与. 2008 年, Bonnamour 等 ${ }^{[41]}$ 以邻酰胺基卤代苯 为原料, 在 $\mathrm{Cs}_{2} \mathrm{CO}_{3} / \mathrm{FeCl}_{3} / \mathrm{TMHD}$ 催化体系作用下，通过 分子内环化反应生成 2-芳基苯并噁唑(表 1), 产率最高 可达 $96 \%$. 由于位阻效应，与噁唑环相连的 $\mathrm{R}^{2}$ (芳基)基 团中邻位有取代基时，产率相对较低. 该方法所用催化 体系廉价易得、催化效率高, 为 2-取代苯并噁唑提供了 一种有价值的合成方法.

Evindar 等 ${ }^{[42]}$ 在 $\mathrm{CuI} / 1,10$-邻菲罗啉催化体系作用下, 以邻酰胺基卤代苯为原料，合成了 2-取代苯并噁唑 (Eq. 33). 卤素的种类对反应有较大影响, 反应速率随着 $\mathrm{I}>\mathrm{Br}>\mathrm{Cl}$ 顺序而降低. 当 $Z$ 为 $S$ 时, 产物是 2-取代苯并 噻唑. Gereon 等 ${ }^{[43]}$ 以邻酰氨基卤代苯为原料, 在 $\mathrm{Cs}_{2} \mathrm{CO}_{3}$ 及 $\mathrm{CuI}$ 作用下，在 $\mathrm{DME}$ 溶剂中, $110{ }^{\circ} \mathrm{C}$ 反应生成 2-乙基 苯并噁唑.

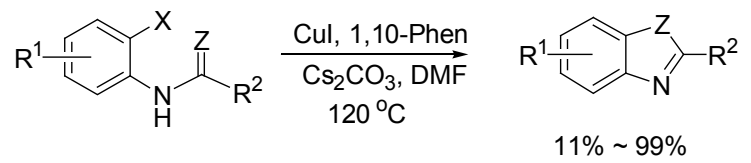

$\mathrm{X}=\mathrm{Cl}, \mathrm{Br}, \mathrm{I} ; \mathrm{Z}=\mathrm{O}, \mathrm{S}$

$\mathrm{R}^{1}=4-\mathrm{Me}, 4-\mathrm{Cl}, 5-\mathrm{CF}_{3}$, etc. $\mathrm{R}^{2}=\mathrm{Ph}, 4-\mathrm{ClC}_{6} \mathrm{H}_{4}, 2-\mathrm{OMeC}_{6} \mathrm{H}_{4}$, etc

近年来，纳米金属化合物由于其良好的催化活性， 受到人们的广泛关注. 2009 年, $\mathrm{Saha}$ 等 ${ }^{[44]}$ 以纳米 $\mathrm{CuO}$ 和 $\mathrm{K}_{2} \mathrm{CO}_{3}$ 为催化剂, 在 DMSO 中反应合成了 2-取代苯并噁 唑(Eq. 34)，产率最高可达 98\%。该方法反应条件温和、 选择性好, 为合成 2-取代苯并䅉唑提供了新方法. 
表 1 铁催化 2-取代苯并噁唑类化合物的合成

Table 1 Iron-catalyzed synthesis of 2-substituted benzoxazole derivatives
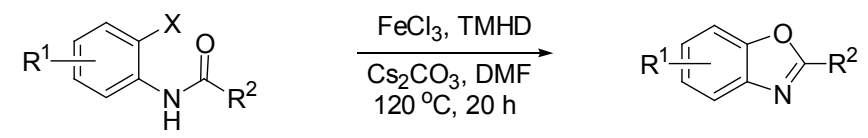

(n)

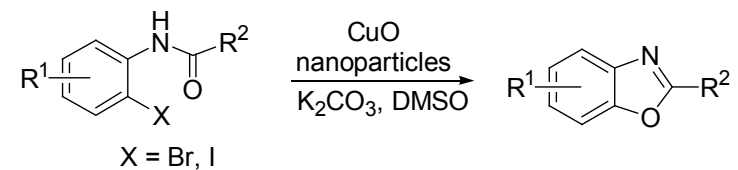

在此基础上, 该课 题 组 ${ }^{[45]}$ 换用钴 (II) 配合物 / 1,10-Phen $/ \mathrm{K}_{2} \mathrm{CO}_{3}$ 催化体系, 使邻卤代酰胺发生分子内 $\mathrm{C}-\mathrm{N}$ 和 $\mathrm{C}-\mathrm{O}$ 的交叉偶联反应, 生成 2-取代苯并噁唑 (Eq. 35), 产率最高可达 $97 \%$.

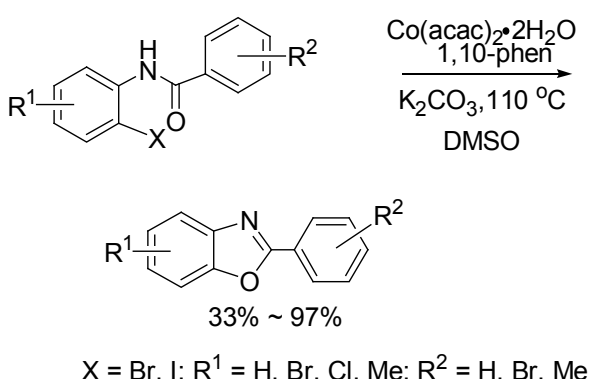

$\mathrm{X}=\mathrm{Br}, \mathrm{I} ; \mathrm{R}^{1}=\mathrm{H}, \mathrm{Br}, \mathrm{Cl}, \mathrm{Me} ; \mathrm{R}^{2}=\mathrm{H}, \mathrm{Br}, \mathrm{Me}$
Viirre 等 ${ }^{[46]}$ 在微波辐射下, 以 $\mathrm{Cs}_{2} \mathrm{CO}_{3} /$ 邻二氮菲 $/ \mathrm{CuI}$ 为催化体系, 邻溴苯胺和酰氯作用生成 2-取代苯并噁唑 (Eq. 36), 产率最高可达 $97 \%$. 在反应过程中, 邻澳苯胺 首先与酰氯作用生成邻溴代酰胺基苯, 再经过环化生成 目标产物. 与传统的加热方法相比, 微波辐射法大大缩 短了反应时间，提高了效率.

$$
\mathrm{R}^{1}=\mathrm{H}, \mathrm{Me}, \mathrm{F}, \text { etc.; } \mathrm{R}^{2}=\mathrm{Ph}, 2-\mathrm{MePh}, 2 \text {-thienyl, } n-\mathrm{C}_{11} \mathrm{H}_{23} \text {, etc. }
$$

Ueda 等 ${ }^{[47]}$ 以苯甲酰胺基苯为原料, 在 $\mathrm{Cu}(\mathrm{OTf})_{2} / \mathrm{O}_{2}$ 催化体系作用下, $140{ }^{\circ} \mathrm{C}$ 时发生环化反应生成 2-苯基苯 并噁唑(Eq. 37), 产率最高可达 93\%. 


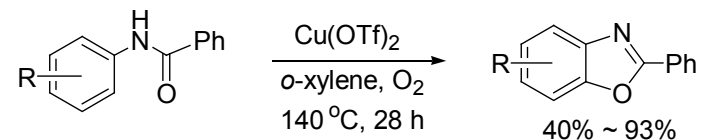

$\mathrm{R}=\mathrm{Me}, \mathrm{OEt}, \mathrm{Ph}, \mathrm{CO}_{2} \mathrm{Me}, \mathrm{COPh}, \mathrm{Cl}$, etc.

\section{2 由其他取代酰胺基苯合成 2-取代苯并噁唑}

2013 年, Sedelmeier 等 ${ }^{[48]}$ 用 $n$-BuLi 处理 3-卤代- $N$ 酰基苯胺, 在连续反应器内经过强碱脱除质子和分子内 环化等一系列过程合成了 2-取代苯并啞唑(Eq. 38). 采 用连续流动技术能够精确地控制反应温度、及时淬灭反 应和最大限度地减少副反应的发生，提高原子经济性， 符合绿色化学的发展理念.

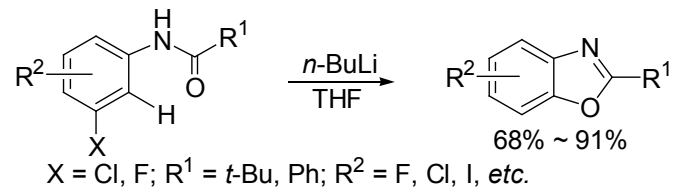

除邻卤代酰胺基苯外, 邻酰胺基苯酚也可以作为起 始原料合成 2-取代苯并噁唑. 例如, 2013 年 Suresh 等 ${ }^{[49]}$ 以邻酰胺基苯酚为原料, 在蒙脱土 $\mathrm{K} 10$ 催化下, 通过环 化生成 2-取代苯并噁唑(Eq. 39), 产率最高可以达到 $98 \%$. 催化剂蒙脱土 K10 可以重复利用 5 次而产率保持 相对稳定.

$$
\mathrm{R}^{1}=\mathrm{H}, \mathrm{Cl}, \mathrm{SO}_{2} \mathrm{Et} \text {, etc.; } \mathrm{R}^{2}=\mathrm{Me}, \mathrm{Ar}, \mathrm{Py} \text {, etc. }
$$

\section{3 苯并噁唑与其他基团偶联合成 2-取代苯并噁} 唑

近年来, 利用钯、铜等金属的催化作用, 通过偶联 反应直接合成 2-取代苯并噁唑的报道越来越多. 该方法 效率高、选择性好, 是近几年发展起来的合成 2-取代苯 并噁唑的重要方法.

\section{1 苯并噁唑与芳环偶联合成 2-取代苯并噁唑}

2012 年, $\mathrm{Wu}$ 等 ${ }^{[50]}$ 利用 $\mathrm{Pd}(\mathrm{OAc})_{2} / \mathrm{CuBr}_{2}$ 活化苯并噁 唑与苯中的 $\mathrm{C}-\mathrm{H}$ 键, 使其发生分子间偶联反应生成 2芳基苯并噁唑(Eq. 40), 产率最高可达 94\%. 其中 $\mathrm{CuBr}_{2}$ 作为该反应的添加剂, 既可以活化苯并噁唑, 还可以避 免催化剂中毒.

2012 年, $\mathrm{Mao}$ 等 ${ }^{[51]}$ 在 $\mathrm{Cu}(\mathrm{OAc})_{2}$ 的催化下, 通过两种 芳杂环间发生偶联反应得到 2-杂环取代的苯并噁唑(Eq. 41). 反应通过铜的还原反应 $[\mathrm{Cu}(\mathrm{II})$ 变为 $\mathrm{Cu}(0)]$ 实现, 在 反应中观察到 “铜镜” 生成. 该方法操作简洁而且绿色

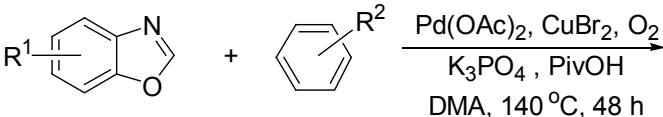

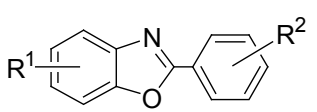

$$
\begin{aligned}
& 43 \% \text { } 94 \% \\
& \mathrm{R}^{1}=\mathrm{CH}_{3}, \mathrm{~F}, \mathrm{Cl}, \mathrm{OMe}, \mathrm{CO}_{2} \mathrm{Me}, \mathrm{Ph} ; \mathrm{R}^{2}=\mathrm{Me}, \mathrm{Cl}, \mathrm{CO}_{2} \mathrm{Me}
\end{aligned}
$$

环保，是合成非对称双杂环化合物的好方法.

$$
\begin{aligned}
& \mathrm{X}=\mathrm{O}, \mathrm{S}, \mathrm{NR}_{3} ; \mathrm{R}^{1}=\mathrm{CH}_{3}, \mathrm{Cl} ; \mathrm{R}^{2}=\mathrm{OCH}_{3}, \mathrm{Ph}, 4-\mathrm{ClC}_{6} \mathrm{H}_{4} \text {, etc. }
\end{aligned}
$$

\section{2 苯并噁唑通过 Heck 反应和 Suzuki 反应生成 2-取 代苯并噁唑}

2013 年, $\mathrm{Yu}$ 等 ${ }^{[52]}$ 以苯并啞唑和多氟代苯为原料, 在 $\mathrm{Pd}\left(\mathrm{CH}_{3} \mathrm{CN}\right)_{2} \mathrm{Cl}_{2} / \mathrm{DPPBz} / \mathrm{LiO} t-\mathrm{Bu}$ 催化体系作用下，通过 活化 $\mathrm{C}-\mathrm{F}$ 和 $\mathrm{C}-\mathrm{H}$ 使之发生 Heck 偶联反应，生成 2芳基苯并惡唑(Eq. 42). 研究发现，当邻位的 “方向基团 (directing group)” 是 2-吡啶基时产率最高(93\%).

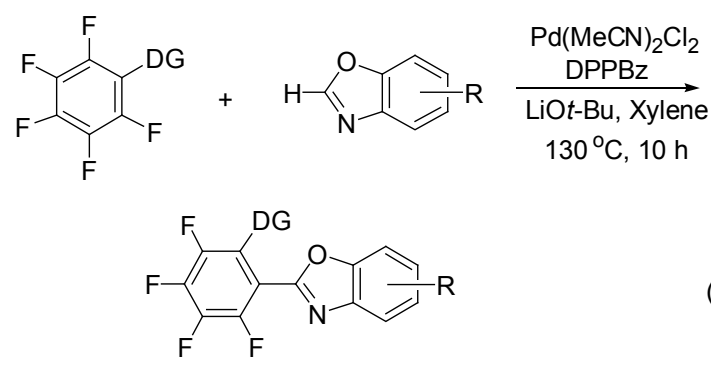

$$
\mathrm{DG}=\mathrm{N}_{\mathrm{N}}=\mathrm{H}, \mathrm{Me}, \mathrm{Cl}, t-\mathrm{Bu}, \mathrm{Ph}, p \text {-tolyl, } p \text { - } \mathrm{MeOC}_{6} \mathrm{H}_{4}
$$

2011 年, Zhang 等 ${ }^{[53]}$ 以纳米 $\mathrm{CuO}$ 为催化剂, 通过苯 并噁唑和碘代苯发生偶联反应生成 2-芳基苯并噁唑(Eq. 43), 产率高达 93\%. 催化剂纳米 $\mathrm{CuO}$ 可以多次重复使 用而活性不会降低，以一种理想的催化剂。

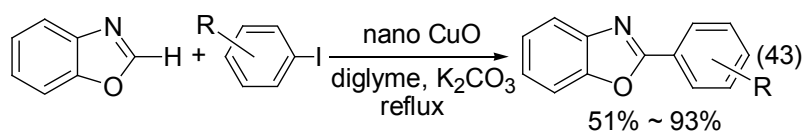

$\mathrm{R}=\mathrm{H}, \mathrm{CH}_{3}, \mathrm{CN}, \mathrm{Cl}, \mathrm{COOEt}, \mathrm{OCH}_{3}$

上述方法不仅适用于卤代芳烃，脂肪基卤也可以发 生类似的偶联反应. Gerelle 等 ${ }^{[54]}$ 在 $\mathrm{Pd}(\mathrm{dppf}) \mathrm{Cl}_{2} / \mathrm{PPh}_{3} /$ $\mathrm{Ag}_{2} \mathrm{CO}_{3}$ 催化体系作用下, 通过烯基碘化物与苯并噁唑 
之间的交叉偶联反应，生成 2-取代苯并噁唑(Eq. 44). 值 得关注的是, 碘代烯烃的几何构型在整个偶联过程中保 持不变. 该反应在水中进行, 条件温和, 效率高, 是一 种绿色环保的好方法.

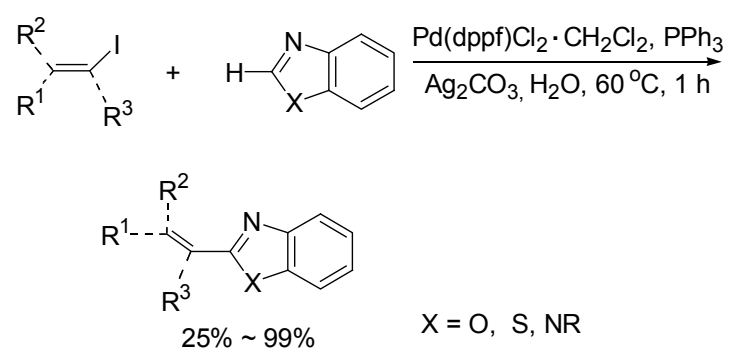

2011 年, Ranjit 等 ${ }^{[55]}$ 选择 $\mathrm{Pd}(\mathrm{OAc})_{2} / \mathrm{Cu}(\mathrm{OAc})_{2} / 1,10-$ phenanthroline 组成催化体系, 以 $\mathrm{K}_{3} \mathrm{PO}_{4}$ 为缚酸剂, 苯并 噁唑和芳基硼酸通过 Suzuki 偶联反应生成 2-芳基苯并 噁坐(Eq. 45), 产率最高为 $90 \%$. 该方法的适用范围还可 进一步扩展到 2-芳基苯并噻唑的合成.

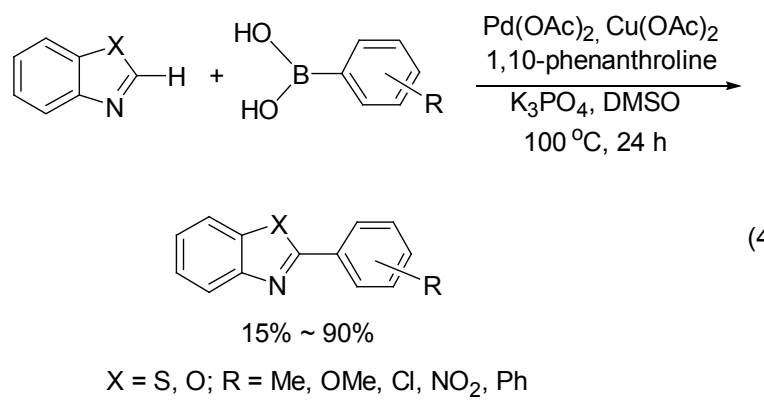

\section{3 苯并噁唑与羧酸或 $\mathrm{CO}_{2}$ 偶联合成 2-取代苯并噁唑}

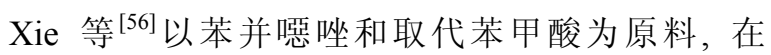
$\mathrm{PdCl}_{2} / \mathrm{PPh}_{3}$ 体系催化下, 通过偶联脱羧生成 2-芳基苯并 啞唑(Eq. 46). 研究发现, 无论苯甲酸的苯环上连有吸电 子基团还是供电子基团, 反应均能够顺利发生. 由于反 应过程有 $\mathrm{CO}_{2}$ 放出, 反应几乎可以定量进行.
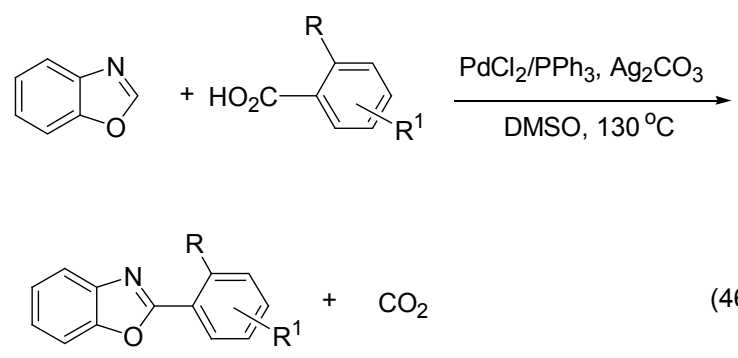

2012 年, Inomata 等 ${ }^{[57]}$ 通过 1,2,3-三唑-5-亚铜活化啞 唑环的 $\mathrm{C}-\mathrm{H}$ 键, 使苯并噁唑与 $\mathrm{CO}_{2}$ 偶联, 并进一步与 碘甲烷(溶于 DMF 中)作用生成 2-甲酸甲酯基苯并噁唑 (Eq. 47). 该方法产率高, 操作简单, 同样适用于 2-取代
苯并噻唑的合成.

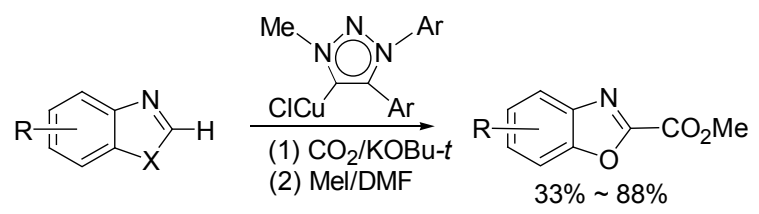

$X=\mathrm{O}, \mathrm{S} ; \mathrm{R}=5-\mathrm{Me}, 5-\mathrm{NO}_{2}, 5-\mathrm{Cl}$, etc.

\section{4 苯并噁唑与醛偶联合成 2-取代苯并噁唑}

Teo 等 ${ }^{[58}$ 通过单质碘催化苯并噁唑和苯甲醛间的偶 联反应，合成了 2-芳基苯并噁唑(Eq. 48), 产率最高可达 $81 \%$ ，该方法简单易行，用单质碘代替金属化合物引发 偶联反应，是很有意义的尝试.

$$
\text { metal-free }
$$

该反应的历程如下: 苯并噁唑在 $I_{2}$ 作用下氧化开环 形成邻氨基苯酚 $\mathbf{g}, \mathbf{g}$ 通过两种可能的途径(A 和 B)形成 Schiff 碱 $\mathbf{i}$. 其中, 途径 A 是 $\mathbf{g}$ 直接与芳醛发生缩合反应 生成 Schiff 碱 $\mathbf{i}$; 途径 $\mathrm{B}$ 是 $\mathbf{g}$ 先与苯并噁唑反应形成中 间体 $\mathbf{h}, \mathbf{h}$ 再与苯甲醛反应脱去一分子苯并啞唑形成 Schiff 碱 i. 最后, $\mathbf{i}$ 在 $\mathrm{I}_{2}$ 催化下环化形成产物 2-苯基苯 并噁唑(Scheme 8).

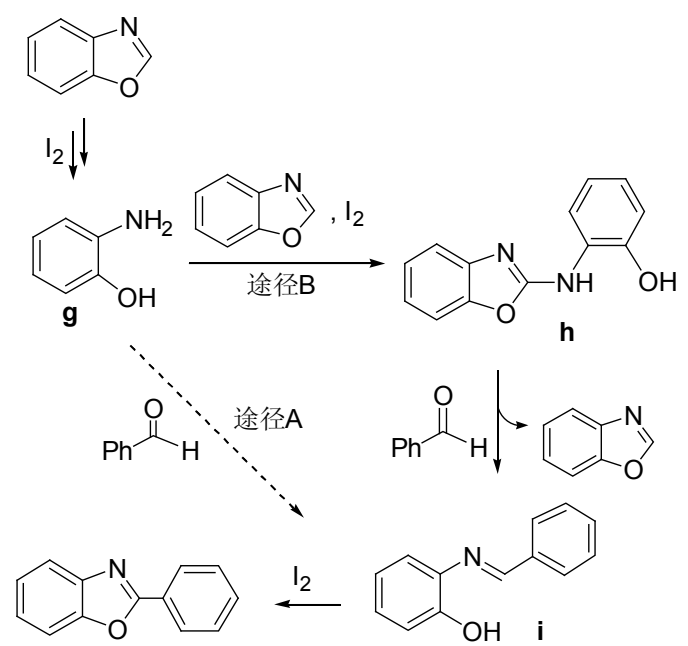

Scheme 8

\section{5 苯并噁唑与胺偶联合成 2-取代苯并噁唑}

$\mathrm{Xu}$ 等 ${ }^{[59]}$ 采用苯并啞唑和仲胺作用, 在 Fenton 试剂 $\left(\mathrm{FeCl}_{2} / \mathrm{H}_{2} \mathrm{O}_{2}\right)$ 的氧化下合成了 2-仲氨基苯并噁唑，产率 最高可达 $97 \%$. 反应过程中首先是原料中的噁唑开环, 
与仲氨反应生成榺类化合物, 然后在 Fenton 试剂作用下 关环得到 2-仲氨基苯并噁唑(Eq. 49). 该方法在反应过 程中, 使用无毒价廉的 $\mathrm{FeCl}_{2} / \mathrm{H}_{2} \mathrm{O}_{2}$ 氧化体系, 是一种高 效、环境友好的苯并噁唑仲氨基化方法.

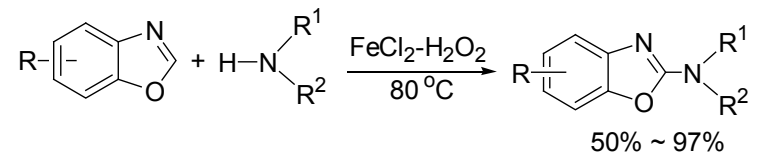

Guo 等 ${ }^{[60]}$ 以 $\mathrm{CuBr}_{2} \mathrm{CH}_{3} \mathrm{COOH}$ 组成催化体系, 在氧 气气氛中, 通过苯并噁唑和叔胺作用生成 2-仲氨基苯并 啞唑和醛(Eq. 50), 产率最高可达 99\%. 该方法采用 $\mathrm{O}_{2}$ 为氧化剂, 叔胺作为氮源, 方法新颖, 催化剂廉价易得, 为形成 $\mathrm{C}-\mathrm{N}$ 键开辟了一种新的途径.

$$
\text { 事 }
$$

Chen 等 ${ }^{[61]}$ 以 $\mathrm{K}_{2} \mathrm{Cr}_{2} \mathrm{O}_{7}$ 为氧化剂, 在 bipyPdCl 2 / $\mathrm{CuCN} / \mathrm{K}_{2} \mathrm{CO}_{3}$ 催化下, 通过苯并噁唑和胺基磺酰氯作用 生成 2-胺代苯并啞唑(Eq. 51), 产率高达 91\%.

$$
\text { }
$$

2013 年, Inomata 等 ${ }^{[62]}$ 在氮杂环卡宾铜(IPr)CuI/ $\mathrm{K}_{2} \mathrm{CO}_{3}$ 催化下苯硫酚与苯并噁唑发生硫醚化偶联反应, 生成了 2-芳硫代苯并噁唑(Eq. 52), 产率为 $22 \% \sim 82 \%$. 该方法也同样适用于 2-芳硫代苯并噻唑的合成.

$$
\begin{aligned}
& \mathrm{R}=\mathrm{C}_{6} \mathrm{H}_{5}, 4-\mathrm{MeC}_{6} \mathrm{H}_{4}, 2-\mathrm{MeC}_{6} \mathrm{H}_{4} \text {, etc. } \\
& \mathrm{R}^{1}=\mathrm{H}, \mathrm{Me}, \mathrm{MeO}, \mathrm{Cl}, \mathrm{Br}, \mathrm{NO}_{2}
\end{aligned}
$$

\section{4 邻二卤代苯与酰胺作用合成 2-取代苯并噁唑}

2008 年, Viirre 等 ${ }^{[63]}$ 在 CuI-DMEDA(二甲基乙二胺) 体系催化下，1,2-二溴苯和酰胺发生交叉偶合反应生成 2-取代苯并噁唑(Eq. 53), 产率为 56\% 77\%. 该反应简 单易行, 是合成 2-取代苯并噁唑类化合物行之有效的好 方法.

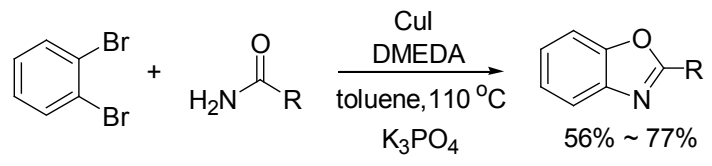

$\mathrm{R}=\mathrm{Ph}, 2-\mathrm{MeC}_{6} \mathrm{H}_{4}, 2$-thienyl, $n-\mathrm{C}_{11} \mathrm{H}_{23}$

2013 年, $\mathrm{Wu}$ 等 ${ }^{[64]}$ 采用溴苯与 $\mathrm{NH}_{3}$ 和 $\mathrm{CO}$ 作用生成 酰胺中间体，1,2-二溴苯再与酰胺中间体在 $\mathrm{CuI} /$ DMEDA $/ \mathrm{K}_{2} \mathrm{CO}_{3}$ 体系催化下反应，合成了 2-芳基苯并噁 唑(Scheme 9), 产率为 39\% 71\%.
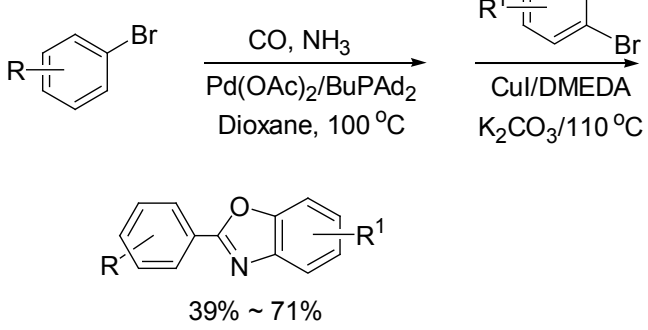

Scheme 9

\section{5 由肟类化合物合成 2-取代苯并噁唑类化合物}

2010 年, Gadakh 等 ${ }^{[65]}$ 采用肜类化合物在 $\mathrm{POCl}_{3}$ 的作 用下，经重排、环化缩合生成 2-取代苯并噁唑类化合物 (Eq. 54), 产率为 $60 \% \sim 70 \%$, 所得产物具有优良的抗微 生物活性。
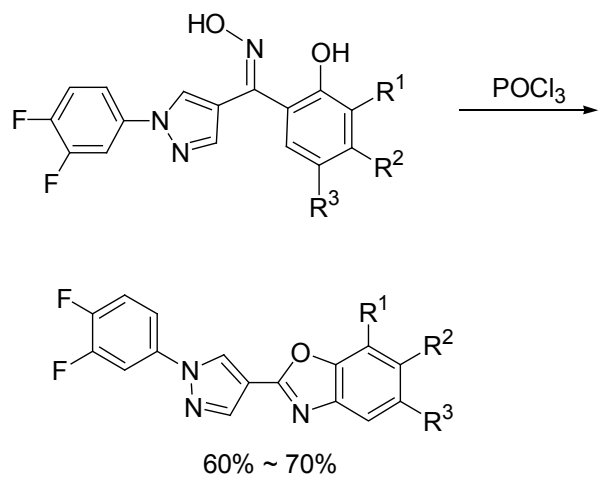

2011 年, Guru 等 ${ }^{[66]}$ 以二芳甲醛肟醚为原料，以 $\mathrm{Cu}(\mathrm{OTf})_{2}$ 为催化剂, 在氧气气氛中, 经分子内重排、环 化生成 2-芳基取代苯并啞唑(Eq. 55)，产率 80\% 90\%. 整个反应过程涉及 $\mathrm{C}-\mathrm{H}$ 的功能化以及 $\mathrm{C}-\mathrm{N} / \mathrm{C}-\mathrm{O}$ 化 学键的形成.

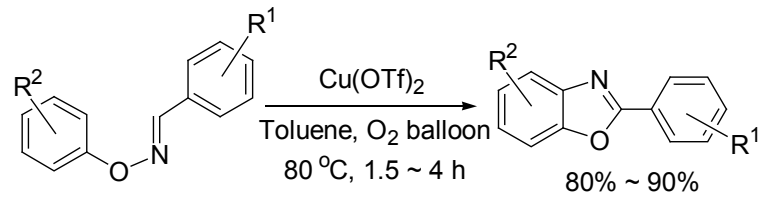

$\mathrm{R}^{1}, \mathrm{R}^{2}=\mathrm{EWG}, \mathrm{EDG}$ 


\section{6 由其他原料合成 2-取代苯并噁唑}

2008 年, Marsden 等 ${ }^{[67]}$ 以有机膦试剂为催化剂, 通 过邻异氧酸酯基苯甲酸酚酯发生分子内 “氮杂-Wittig 环 化” 反应，合成 2-苯基苯并噁唑(Eq. 56). 该方法采用的 有机膦试剂催化效率高而且可以循环使用, 为合成 2-取 代苯并啞唑提供了一种新型的非金属催化剂.
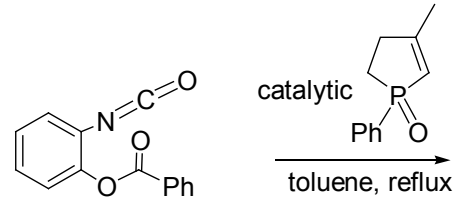

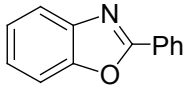

5 mol $\%$ cat. : $87 \%$ $1 \mathrm{~mol} \%$ cat. : $75 \%$
2012 年, Okitsu 等 ${ }^{[68]}$ 以邻异氰基苯基缩酮化合物为 原料，在 $\mathrm{BF}_{3} \cdot \mathrm{OEt}_{2} / 2,4,6$-三甲基吡啶催化体系作用下, 通过烷氧甲酰化反应合成了 2-烷氧基苯并惡唑(Eq. 57), 产率最高可达 $96 \%$. 反应过程经过基团的串联迁移得以 实现.

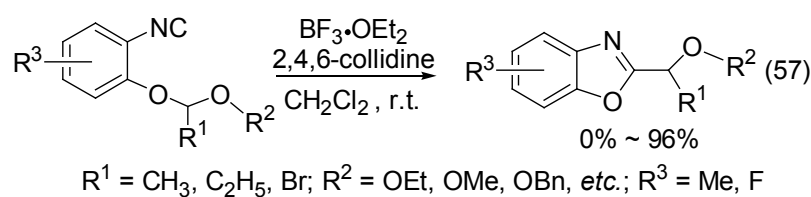

2011 年, Chen 等 ${ }^{[69]}$ 以取代邻亚胺基丙基苯酚为原 料, 合成了 2-乙基苯并噁唑(Eq. 58). 反应条件对产物结 构有重要影响: 在 $\mathrm{NaOCl}$ 存在下, 芳基上的基团发生 $[1,2]$ 迁移, 生成 2-乙基苯并噁唑; 而在 THF 中则生成 3乙基苯并异噁唑. 此外, 苯环上的电子云排布对产物结 构也有影响: 富电子的芳环有利于贝克曼重排生成 2-乙 基苯并噁唑, 而缺电子的芳环则有利于形成 $\mathrm{N}-\mathrm{O}$ 键, 得到 3-乙基苯并异噁唑.<smiles>CCC(=N)c1cc(Cl)c(C)cc1O</smiles><smiles>CCc1nc2cc(Cl)c(C)cc2o1</smiles>

\section{7 结语及展望}

综上所述，随着 2-取代苯并噁唑的应用范围越来越 广泛，有关其合成方法的报道逐年增多. 本文分别以邻 氨基苯酚、取代酰胺基苯、苯并噁唑、邻二卤代苯与酰 胺、肟类化合物以及其他物质等 2-取代苯并噁唑的合成 中所选用的不同起始原料为基准进行分类, 对 2-取代苯 并噁唑的合成方法进行了归纳总结. 文中所提及的各种 方法优势互补, 适用于不同反应原料及条件下 2-取代苯 并腎唑类化合物的合成.
随着 2-取代苯并噁唑类化合物的合成和应用研究 的不断深入, 高耗能、高污染的传统合成方法, 逐渐被 高效、绿色环保的合成方法如微波促进、无溶剂合成、 固相合成以及非金属催化等所替代，这也是 2-取代苯并 噁唑类化合物合成方法的发展趋势. 相信在广大化学工 作者的共同努力下，还会有越来越多的新方法和新手段 会不断应用于 2-取代苯并噁唑类化合物的合成中.

\section{References}

[1] Razavi, H.; Palaninathan, S. K.; Powers, E. T. Angew. Chem., Int. Ed. 2003, 42, 2758.

[2] (a) Gao, R.; Zhang, M.; Liang, T.; Wang, F.; Sun, W.-H. Organometallics 2008, 27, 5641.

(b) Gao, R.; Li, Y.; Wang, F.; Sun, W.-H. Eur. J. Inorg. Chem. 2009, 4149.

[3] Ge, F.; Wang, Z.; Wan, W.; Lu, W.; Hao, J. Tetrahedron Lett. 2007, $48,3251$.

[4] Zhang, H. Z.; Zhou, C. H.; Geng, R. X.; Ji, Q. G. Chin. J. Org. Chem. 2011, 31, 1963 (in Chinese). (张慧珍, 周成合, 耿蓉霞, 吉庆刚, 有机化学, 2011, 31, 1963.)

[5] Kawashita, Y.; Nakamichi, N.; Kawabata, H.; Hayashi, M. Org. Lett. 2003, 5, 3713.

[6] Haneda, S.; Gan, Z. B.; Eda, K.; Hayashi, M. Organometallics 2007, 26, 6551.

[7] Xiao, L. W. Chin. J. Org. Chem. 2011, 31, 878 (in Chinese). (肖立伟, 有机化学, 2011, 31, 878.)

[8] Cao, K.; Tu, Y. Q.; Zhang, F. M. Sci. China Chem. 2010, 53, 130.

[9] Inamdar, S. M.; More, V. K.; Mandal, S. K. Tetrahedron Lett. 2013, $54,579$.

[10] Shavaleev, N. M.; Scopelliti R.; Gumy, F.; Bunzli, J. G. Inorg. Chem. 2009, 48, 6178.

[11] Heravi, M. M.; Sadjadi S.; Oskooie, H. A.; Shoar, R. H.; Bamoharram, F. F. J. Chin. Chem. Soc. 2008, 55, 890.

[12] Kumar, A.; Maurya, R. A.; Ahmad, P. J. Comb. Chem. 2009, 11, 198.

[13] Firouz, M. M.; Ghasem, R. B.; Hossein, I. Synth. Commun. 2006, $36,2543$.

[14] Balaswamy, G.; Srinivas K.; Pradeep P.; Sarangapani M. Int. J. Chem. Sci. 2012, 10, 619.

[15] Ertan, T.; Yildiz, I.; Tekiner-Gulbas, B.; Bolelli, K.; Temiz-Arpaci, O.; Ozkan, S.; Kaynak, F.; Yalcin, I.; Aki. E. Eur. J. Med. Chem. 2008, 1.

[16] Wei, C. X.; Wu, D.; Sun, Z. G.; Chai, K. Y.; Quan, Z. S. Med. Chem. Res. 2010, 19, 925.

[17] Soares, A. M. S.; Costa, S. P. G.; Goncalves, M. S. T. Tetrahedron 2010, 66, 8189.

[18] Zhou, H. J.; Wang, L. G.; Yin, B. S. Chem. Res. 2003, 14, 39 (in Chinese).

(周红军, 王立格, 尹帮少, 化学研究, 2003, 14, 39.)

[19] Heravi, M. M.; Sadjadi, S.; Oskooie, H. A.; Shoar, R. H.; Bamoharram, F. F. J. Chin. Chem. Soc. 2008, 55, 890.

[20] Boyle, K. E.; MacMillan, K. S.; Ellis, D. A.; Lajiness, J. P.; Robertson, W. M.; Boger, D. L. Bioorg. Med. Chem. Lett. 2010, 20, 1854.

[21] Radi, M.; Saletti, S.; Botta, M. Tetrahedron Lett. 2008, 49, 4464.

[22] Xiao, L.-W.; Zhang, M.; Sun, W.-H. Chem. Res. Chin. Univ. 2010, 25,1

[23] Mohammadpoor-Baltork, I.; Moghadam, M.; Tangestaninejad, S.; Mirkhani, V.; Zolfigol, M. A.; Hojati, S. F. J. Iran. Chem. Soc. 
2008, 5, 65 .

[24] Lim, H. J.; Myung, Do.; Lee, Y. C.; Jung M. H. J. Comb. Chem. 2008, 10, 501 .

[25] Bastug, G.; Eviolitte, C.; Markó, I. E. Org. Lett. 2012, 14, 3502.

[26] Jeyanthi, P.; Pazhanisamy, P. Int. J. ChemTech Res. 2010, 2, 1170.

[27] Christina, P.; Stella, R.; Rajam, S.; Venkatraman, B. R. J. Chem. Pharm. Res. 2012, 4, 2988.

[28] Pottorf, R. S.; Chadha, N. K.; Katkevics, M.; Ozola, V.; Suna, E.; Ghane, H.; Regberg, T.; Player, M. R. Tetrahedron Lett. 2002, 44, 175.

[29] Shoar, R. H.; Heidary, M.; Farzaneh, M.; Malakouti, R. Synth. Commun. 2009, 39, 1742.

[30] Nadaf, R. N.; Siddiqui, S. A.; Lahoti, R. J. J. Mol. Catal. A: Chem. 2004, 214, 155.

[31] Chun, Y. S.; Lim, S. J.; Oh, S. J.; Moon, D. H.; Kim, D. J.; Cho, C. G.; Yoo, K. H. Bull. Korean Chem. Soc. 2008, 29, 1765.

[32] Gao, R.; Xiao, L.-W.; Hao, X.; Sun, W.-H.; Wang, F. S. Dalton Trans. 2008, 5645.

[33] Gu, L. J.; Jin, C.; Guo, J. M.; Zhang, L. Z.; Wang, W. Chem. Commun. 2013, 49, 10968.

[34] Wilfred, C. D.; Taylor, R. J. K. Synlett 2004, 1628.

[35] Wu, M.; Hu, X.; Liu, J.; Liao, Y.; Deng, G.-J. Org. Lett. 2012, 14, 2722.

[36] Endo, Y.; Bäckvall, J. E. Chem.-Eur. J. 2012, 43, 13609.

[37] Jin, X.; Liu, Y.; Lu, Q.; Yang, D.; Sun, J.; Qin, S.; Zhang, J.; Shen, J.; Chu, C.; Liu, R. Org. Biomol. Chem. 2013, 11, 3776.

[38] Nguyen, T. B.; Ermolenko, L.; Dean, W. A.; Al-Mourabit, A. Org. Lett. 2012, 14, 5948.

[39] Boissarie, P. J.; Hamilton, Z. E.; Lang, S.; Murphy, J. A.; Suckling, C. J. Org. Lett. 2011, 13, 6256.

[40] Zhang, C. R.; Wang, L.; Ge, Y. L.; Ju, X. L. Chin. J. Org. Chem. 2007, 27, 1432 (in Chinese). (张成仁, 王柳, 葛燕丽, 巨修练, 有机化学, 2007, 27, 1432.)

[41] Bonnamour, J.; Bolm, C. Org. Lett. 2008, 10, 2665.

[42] Evindar, G.; Batey, R. A. J. Org. Chem. 2006, 71, 1802.

[43] Gereon, A.; Frank, G. A. Adv. Synth. Catal. 2004, 346, 1661.

[44] Saha, P.; Ramana, T.; Purkait, N.; Ali, M. A.; Paul, R.; Punniyamurthy, T. J. Org. Chem. 2009, 74, 8719.

[45] Saha, P.; Ali, M. A.; Ghosh, P.; Punniyamurthy, T. Org. Biomol. Chem. 2010, 8, 5692.

[46] Viirre, R. D.; Evindar, G.; Batey, R. A. J. Org. Chem. 2008, 73, 3452 .
[47] Ueda, S.; Nagasawa, H. Angew. Chem., Int. Ed. 2008, 47, 6411.

[48] Sedelmeier, J.; Lima, F.; Litzler, A.; Martin, B.; Venturoni, F. Org. Lett. 2013, 15, 5546.

[49] Suresh, D.; Dhakshinamoorthy, A.; Pitchumani, K. Tetrahedron Lett. 2013, 54, 6415.

[50] Wu, G.; Zhou, J.; Zhang, M.; Hu, P.; Su, W. Chem. Commun. 2012, $48,8964$.

[51] Mao, Z.; Wang, Z.; Xu, Z.; Huang, F.; Yu, Z.; Wang, R. Org. Lett. 2012, 14, 3854.

[52] Yu, D.; Lu, L.; Shen, Q. Org. Lett. 2013, 15, 940.

[53] Zhang, W.; Zeng, Q.; Zhang, X.; Tian, Y.; Yue, Y.; Guo, Y.; Wang, Z. J. Org. Chem. 2011, 76, 4741.

[54] Gerelle, M.; Dalencon, A. J.; Willis, M. C. Tetrahedron Lett. 2012, 53, 1954.

[55] Ranjit, S.; Liu, X. G. Chem. Eur. J. 2011, 17, 1105.

[56] Xie, K.; Yang, Z.; Zhou, X.; Li, X. ; Wang, S.; Tan, Z.; An, X.; Guo, C. Org. Lett. 2010, 12, 1564.

[57] Inomata, H.; Ogata, K.; Fukuzawa, S.; Hou, Z. M. Org. Lett. 2012, 14, 3986.

[58] Teo, Y. C.; Riduan, S. N.; Zhang, Y. G. Green Chem. 2013, 15, 2365.

[59] Xu, D.; Wang, W.; Miao, C.; Zhang, Q.; Xia, C.; Sun, W. Green Chem. 2013, 15, 2975.

[60] Guo, S.; Qian, B.; Xie, Y.; Xia, C.; Huang, H. Org. Lett. 2011, 13, 522.

[61] Chen, S.; Zheng, K.; Chen, F. Tetrahedron Lett. 2012, 53, 6297.

[62] Inomata, H.; Toh, A.; Mitsui, T.; Fukuzawa, S. I. Tetrahedron Lett. 2013, 54, 4729.

[63] Viirre, R. D.; Evindar, G.; Batey, R. A. J. Org. Chem. 2008, 73, 3452.

[64] Wu, X.-F.; Neumann, H.; Neumann, S.; Beller, M. Tetrahedron Lett. 2013, 54, 3040 .

[65] Gadakh, A. V.; Pandit, C.; Rindhe, S. S.; Karale, B. K. Bioorg. Med. Chem. Lett. 2010, 20, 5572.

[66] Guru, M. M.; Ali, M. A.; Punniyamurthy, T. Org. Lett. 2011, 13, 1194.

[67] Marsden, S. P.; McGonagle, A. E.; McKeever-Abbas, B. Org. Lett. 2008, 10, 2589.

[68] Okitsu, T.; Nagase, K.; Nishio, N.; Wada, A. Org. Lett. 2012, 14, 708.

[69] Chen, C.-Y.; Andreani, T.; Li, H. Org. Lett. 2011, 13, 63. 